\title{
Brainstem Substrates of Sympatho-Motor Circuitry Identified Using Trans-Synaptic Tracing with Pseudorabies Virus Recombinants
}

\author{
Ilan A. Kerman ${ }^{1,2,3}$ Lynn W. Enquist, ${ }^{4}$ Stanley J. Watson, ${ }^{3}$ and Bill J. Yates ${ }^{1,2}$ \\ Departments of ${ }^{1}$ Neuroscience and ${ }^{2}$ Otolaryngology, University of Pittsburgh, Pittsburgh, Pennsylvania $15213,{ }^{3}$ Mental Health Research Institute, \\ Department of Psychiatry, University of Michigan, Ann Arbor, Michigan 48109, and ${ }^{4}$ Department of Molecular Biology, Princeton University, Princeton \\ New Jersey 08544
}

\begin{abstract}
Previous physiological investigations have suggested the existence of a neural circuit that coordinates activation of motor and autonomic efferents before or at the onset of exercise. Traditionally these circuits have been postulated to involve forebrain areas. However, overlapping populations of medullary reticular formation neurons that participate in motor or autonomic control have been described previously, suggesting that individual pontomedullary reticular formation neurons may coordinate both motor and autonomic responses. We tested this hypothesis by conducting transneuronal retrograde tracing of motor and sympathetic nervous system pathways in rats using recombinant strains of pseudorabies virus (PRV). A PRV strain expressing the green fluorescent protein (PRV-152) was injected into the left gastrocnemius muscle, which was surgically sympathectomized, whereas another recombinant (PRV-BaBlu) was injected into the left adrenal gland. Immunofluorescence methods using monospecific antisera and distinct fluorophores identified neurons infected with one or both of the recombinants. Brainstem neurons coinfected with both PRV recombinants, which presumably had collateralized projections to both adrenal sympathetic preganglionic neurons and gastrocnemius motoneurons, were observed in several areas of the pontomedullary reticular formation. The largest number of such neurons was located in the rostral ventromedial medulla within the ventral gigantocellular nucleus, gigantocellular nucleus pars alpha, raphe obscurus, and raphe magnus. These neurons are candidates for relaying central command signals to the spinal cord.
\end{abstract}

Key words: pseudorabies virus; rats; dual-labeling immunofluorescence; central command; stress; exercise; motor pathway; sympathetic nervous system; raphe nuclei; pontomedullary reticular formation; rostral ventromedial medulla

\section{Introduction}

A wide range of human and animal behavior is characterized by coordinated and complementary activation of motor and autonomic outflows. These behaviors are often part of a broader response to a perturbation in the organism's external environment, such as exposure to a noxious stimulus or encountering a predator or rival and preparing to fight or flee to ensure survival. Alternatively, some behavioral responses during conditions that are not stressful, such as ingesting a meal or sleep, also require synchronized activation of motor and autonomic efferents. Rapid eye movement sleep is characterized by postural muscle atonia that accompanies sympathetic nervous system hypoactivity. Locomotion, as well as other forms of exercise, requires rapid and immediate increases in respiratory function, heart rate, stroke volume, cardiac output, peripheral resistance, and blood pressure (Waldrop et al., 1996). Physiologists have debated for more than a century about the origin of the neural inputs that regulate the concomitant motor and autonomic changes during

Received Dec. 26, 2002; revised March 17, 2003; accepted March 17, 2003.

This work was supported by National Institutes of Health Grants R01 DC00693 (B.J.Y.) and NS33506 (L.W.E.). We thank Kristine Cloonan, Ryan Mori, and Jen-Shew Yen for their excellent technical assistance. We are grateful to Dr. Stephen DiCarlo for his advice on surgical sympathectomy. We thank Dr. Pat Card for critical reading of an earlier version of this manuscript and for his advice and encouragement.

Correspondence should be addressed to I. A. Kerman, 205 Zina Pitcher Place, Mental Health Research Institute, Department of Psychiatry, University of Michigan, Ann Arbor, Ml 48109. E-mail: kerman@umich.edu. Copyright @ 2003 Society for Neuroscience $\quad$ 0270-6474/03/234657-10\$15.00/0 exercise (Rowell, 1993; Waldrop et al., 1996). The crux of the debate has centered on whether the autonomic changes, including alterations in ventilatory and circulatory functions, are triggered by feedback inputs from exercising muscle or are elicited instead by the CNS in parallel with muscle contractions (Waldrop et al., 1996). The coordination of synchronous motor and autonomic activity by the nervous system is termed "central command," and several lines of evidence support its existence. For instance, attempted muscle contraction in paralyzed human subjects led to considerable increases in blood pressure and heart rate (Gandevia et al., 1993). Experiments using human and animal subjects also demonstrated that changes in sympathetic nerve activity often precede the onset of muscle contraction during dynamic and isometric exercise (Matsukawa et al., 1991; Vissing et al., 1991; Victor et al., 1995). Taken together, these data suggest the existence of a neural circuit that is dedicated to coordination of motor and autonomic outflows. Such a circuit may act to increase both motor and sympathetic output during locomotion or a stressful event, whereas it serves an opposite function during sleep.

Traditional views on the central control of motor and autonomic efferent systems have been quite disparate. Motor function has long been thought to be primarily under volitional control and mediated essentially via direct corticospinal and rubrospinal projections (Kandel et al., 2000). Descending reticulospinal projections have also been implicated; however, a clear 
functional role for these pathways is yet to be elucidated. Autonomic functions, on the other hand, have been proposed to occur without conscious intervention, hence the name "autonomic" (Langley, 1921; Cannon, 1963), and are thought to be controlled by hypothalamic, pontine, and medullary regions (Loewy, 1990; Benarroch, 1993). Inputs from the cortex, basal ganglia, and midbrain have also been suggested to contribute to autonomic nervous system regulation, yet the functional significance of these connections is not readily apparent (Verberne et al., 1997).

On the basis of physiological evidence suggesting the existence of central command circuits, we hypothesized that there is a neural pathway dedicated to the coordinated activation of motor and autonomic efferents. Because previous anatomic data have suggested that neurons with dual (motor and sympathetic) innervation targets may be located at the pontomedullary level of the brainstem, we focused our analysis on this portion of the nervous system. These experiments were conducted by injecting distinct recombinants of the transneuronal tracer pseudorabies virus (PRV) into the rat gastrocnemius muscle and the adrenal gland, with subsequent determination of the location of brainstem neurons synaptically connected to each target.

\section{Materials and Methods}

All of the procedures regarding animal use in this study conformed to the Guide for the Care and Use of Laboratory Animals (1996, National Academy of Sciences) and were approved by the Institutional Animal Care and Use Committee of the University of Pittsburgh.

\section{Overview of experimental procedures}

In the present studies we used transgenic recombinants of an attenuated PRV strain, PRV-Bartha, for transneuronal tracing of multisynaptic pathways providing inputs to gastrocnemius motoneurons and adrenal sympathetic preganglionic neurons (SPNs). PRV has preferential tropism for axonal terminals (Vahlne et al., 1978, 1980). It is transported in the retrograde direction from the terminals to the cell body where the viral genome is replicated in the nucleus (Enquist et al., 1998). Capsids are assembled and filled with viral DNA in the nucleus, acquire the mature envelope from a late Golgi compartment, and are transported to sites of afferent synaptic contact where cell-to-cell transneuronal transmission of infection occurs (Card et al., 1993; Enquist et al., 1998). The two viral recombinants that we used were PRV-152 and PRV-BaBlu. Both are derived from PRV-Bartha, which is an attenuated form of the parental strain, PRV-Becker. PRV-BaBlu contains the lacZ gene at the gG locus and produces $\beta$-galactosidase ( $\beta$-gal) under the control of the viral gG promoter (Kim et al., 1999). PRV-152 carries the gene coding for enhanced GFP (EGFP) at the gG locus, which is constitutively expressed under control of the cytomegalovirus immediate early promoter (Smith et al., 2000). Previous studies have demonstrated that PRV-152 and PRVBaBlu are transported trans-synaptically in a retrograde manner, similarly to PRV-Bartha, and that the two recombinants are capable of simultaneously coinfecting the same neuronal population (Standish et al., 1995; Billig et al., 1999, 2000, 2001; Smith et al., 2000). Viral recombinants used in the present studies were harvested from pig kidney cell cultures at a titer of $10^{8} \mathrm{pfu} / \mathrm{ml}$. Viral stocks were aliquoted in $50-100 \mu \mathrm{l}$ volumes and stored at $-80^{\circ} \mathrm{C}$. At the times of injection, viral aliquots were removed from the freezer and kept on ice until immediately before injections.

Forty-three male rats (Sprague Dawley, Hilltop, NY) weighing 210$476 \mathrm{gm}$ were used in these studies. Initial experiments were aimed at optimizing viral infection of motor and sympathetic targets in the periphery. In two of the animals, a monosynaptic retrograde tracer, the $\beta$-subunit of cholera toxin (CT $\beta)$, was injected into the gastrocnemius muscle to determine the location and number of motoneurons innervating this hindlimb muscle. In six rats, increasing volumes of PRV-152 were injected into the gastrocnemius muscle to determine the volume of viral injectate that would infect the greatest number of gastrocnemius motoneurons on a consistent basis. In five additional animals, PRV-152 was injected into the gastrocnemius muscle on one side, and PRV-BaBlu was injected into the contralateral muscle to determine whether the antibodies and reagents used in this study specifically localized each of the two recombinants. In another three rats we determined whether PRV injections into gastrocnemius or the adrenal gland resulted in spread of virus to non-target tissues. Finally, in 27 of the animals, PRV-152 was injected into the left gastrocnemius muscle and PRV-BaBlu was injected into the ipsilateral adrenal gland to determine the locations of brainstem neurons that coordinate both motor and sympathetic activity. During all surgeries, anesthesia was induced with $5 \%$ halothane vaporized in $\mathrm{O}_{2}$ and maintained with $1.5-2.5 \%$ concentration. A surgical plane of anesthesia was achieved such that there was no spontaneous movement and there were no withdrawal responses to tail or foot pinch. At the conclusion of the survival period after transport of viral or other neuroanatomical tracers, animals were deeply anesthetized using Equithesin (mixture of $176 \mathrm{mg} / \mathrm{kg}$ chloral hydrate, $40 \mathrm{mg} / \mathrm{kg}$ sodium pentobarbital, and 87 $\mathrm{mg} / \mathrm{kg} \mathrm{MgSO}_{4}$ ) and perfused transcardially with $100-150 \mathrm{ml}$ of saline followed by $400-500 \mathrm{ml}$ of a $4 \%$ paraformaldehyde solution containing $1.4 \%$ l-lysine and $0.2 \%$ sodium meta-periodate (PLP) (McLean and Nakane, 1974).

\section{Mapping of gastrocnemius motoneurons}

The monosynaptic retrograde tracer CT $\beta$ was injected into the left gastrocnemius muscle in two animals to determine the location of motoneurons innervating this muscle. For this purpose, the overlying skin was incised, and the gastrocnemius muscle was bluntly dissected and separated from the adjacent musculature and connective tissue. A $0.25 \%$ CT $\beta$ solution (List Biological, Campbell, CA) was injected directly into the muscle belly through a $10 \mu \mathrm{l}$ Hamilton syringe; $1 \mu \mathrm{l}$ volumes were delivered at 10 sites throughout the muscle on either side of the sural artery and nerve (Greene, 1963). After a 3 d survival period the animals were perfused transcardially with fixative as described above. The lumbar spinal cord was harvested from these animals, postfixed in PLP for 2-24 $\mathrm{hr}$, stored in $30 \%$ sucrose overnight, and then sectioned in the coronal plane at a thickness of 40 or $50 \mu \mathrm{m}$. The tissue was collected in four bins such that the spacing between adjacent sections in a single bin was 160 or $200 \mu \mathrm{m}$.

\section{Use of pseudorabies virus for transneuronal tracing}

In six preliminary experiments, different volumes of PRV-152 were injected into the left gastrocnemius muscle to determine the volume required to produce maximal motoneuronal infection. For this purpose, the muscle was isolated as described above, and a series of up to 30 injections of $1 \mu \mathrm{l}$ volume were made directly into the muscle belly using a Hamilton syringe. In five rats, $30 \mu \mathrm{l}$ injections of PRV-152 were made into their left gastrocnemius muscles, and the same volume of PRV$\mathrm{BaBlu}$ was delivered into the contralateral gastrocnemius muscle. Spinal cord tissue harvested from this group of animals was used to verify specificity of our immunofluorescence detection methods (see below). The potential for spread of virus to non-target tissues was evaluated in three rats, in which as much as $30 \mu \mathrm{l}$ of PRV-152 was applied on top (but not injected into the belly) of the gastrocnemius muscle and $4 \mu \mathrm{l}$ of PRVBaBlu was injected directly over the viscera surrounding the adrenal gland. No motoneuron labeling by PRV-152 was observed in the spinal cord after these injections. Similarly, only a few intermediolateral cell column (IML) neurons were infected by PRV-BaBlu injected adjacent to the adrenal gland in one of these animals, and in the other two cases there was no infection in the spinal cord. Thus, it seems likely that CNS labeling produced by injection of PRV recombinants into gastrocnemius muscle or the adrenal gland was caused by infection of the target neurons and did not result from spread of virus to non-target tissues.

Hindlimb sympathectomy. In addition to motor efferents, the hindlimb is also innervated by sympathetic efferents that project to blood vessels, sweat glands, and other smooth muscle targets (Jänig and McLachlan, 1992). To prevent infection of sympathetic pathways after injection of PRV-152 into gastrocnemius, the hindlimb was surgically sympathectomized. For this purpose the lumbar sympathetic nerve was dissected via a ventral laparotomy and extirpated from the level of the renal artery caudal to the bifurcation of the abdominal aorta. Neural plexuses running 
along the descending aorta and inferior vena cava were also stripped off under microscopic observation using fine forceps. The abdominal aorta and the inferior vena cava were then swabbed with $10 \%$ phenol dissolved in ethylene glycol. Abdominal musculature was closed with sutures, and the overlying skin was closed with surgical staples. In sham animals the lumbar sympathetic nerve was dissected but remained intact, and no phenol was applied to the abdominal aorta or inferior vena cava. Animals recovered 1-7 d after sympathectomy or sham sympathectomy before being injected with PRV.

Double-virus injections. To determine the location of neurons coordinating muscle contraction and autonomic activity, 27 rats received injections of PRV-152 into their left gastrocnemius muscle and injections of PRV-BaBlu into their left adrenal gland. The gastrocnemius muscle was injected with a $30 \mu \mathrm{l}$ total volume of PRV-152, which was divided into multiple injections of $0.5-1.0 \mu \mathrm{l}$. The ipsilateral adrenal gland was dissected via a left flank incision and gently separated from surrounding viscera and fat; the adjacent connective tissue was then retracted to facilitate access to the gland. Two or three injections of PRV-BaBlu were then made into the gland through a glass pipette attached to a Hamilton syringe; the total volume of virus injected was $2-4 \mu \mathrm{l}$. After each injection the gland was swabbed with a cotton-tip applicator to decrease nonspecific viral spread.

In 14 rats both gastrocnemius and the adrenal gland were injected with PRV on the same day. Most of these animals $(n=10)$ survived $96 \mathrm{hr}$ after the injections, whereas the rest of the rats $(n=4)$ survived $120 \mathrm{hr}$ after virus injections. To determine the locations of SPNs, three of the animals that survived $96 \mathrm{hr}$ after virus injections received 0.6 or $1.0 \mathrm{ml}$ intraperitoneal injections of $0.5 \%$ Fluorogold solution (FG; Fluorochrome, Boulder, CO) $5 \mathrm{~d}$ before virus injections. FG labels all of somatic motoneurons and sympathetic preganglionic neurons; it does not interfere with PRV infectivity and propagation when injections of FG and PRV are temporally separated (Papka et al., 1995; Cano et al., 2000)

In another 13 animals, PRV-152 injections into gastrocnemius preceded PRV-BaBlu injections into the adrenal gland by $24 \mathrm{hr}$ to provide for matching of the infection produced by the two recombinants. Three of the rats survived $96 \mathrm{hr}$ after gastrocnemius muscle injections and $72 \mathrm{hr}$ after adrenal gland injections, whereas 10 survived $120 \mathrm{hr}$ after gastrocnemius muscle injections and $96 \mathrm{hr}$ after adrenal gland injections.

\section{Tissue processing}

After transcardial perfusion with PLP (see above), the entire brain was extracted, the thoracic spinal cord was removed in two blocks (T1-T7 and T8-T13), and individual lumbar spinal cord segments were harvested. In some animals the S1 and S2 spinal levels were also removed, and in six cases the cervical cord was extracted as a single block (C1-C8). Brains were postfixed in PLP for 2-4 hr, whereas spinal cord tissue was left in the fixative overnight. Blocks of thoracic and cervical spinal cord tissue were sectioned in the horizontal plane at a thickness of 35 or 40 $\mu \mathrm{m}$, and sections were separated into three bins. Lumbar spinal cord segments were sectioned coronally at 35 or $40 \mu \mathrm{m}$ and collected into three bins, whereas brains were sectioned coronally at a thickness of 40 $\mu \mathrm{m}$ and collected into six bins. Tissue was stored at $-20^{\circ} \mathrm{C}$ in cryoprotectant (30\% sucrose, $30 \%$ ethylene glycol, $1 \%$ polyvinyl-pyrrolidine) until immunohistochemical processing was conducted.

\section{Immunohistochemical procedures}

Tissue was initially rinsed with $0.1 \mathrm{M}$ PBS several times at room temperature. It was then soaked in a $0.5 \%$ sodium borohydrate solution for 10-30 min and washed thoroughly in $0.1 \mathrm{M}$ PBS before sections were incubated in primary antibody solution.

CT $\beta$ was localized using an immunoperoxidase procedure (Hsu et al., 1981 ) with nickel enhancement. This method used goat polyclonal antiCT $\beta$ antibodies (1:50,000; List Biological), a biotinylated donkey antigoat affinity-purified secondary antibody (1:500; Jackson ImmunoResearch Laboratories, West Grove, PA), and avidin-biotin-peroxidase reagents (ABC Elite kit, Vector Laboratories, Burlingame, CA). Diaminobenzidine was used as chromagen, and its visualization was enhanced with nickel by incubating the sections in a $2.5 \%$ nickel sulfate solution.

In initial parametric experiments, neurons infected with PRV-152 or
PRV-BaBlu were identified with immunoperoxidase procedures that used a rabbit polyclonal antibody (Rb-133, concentration of 1:10,000) that recognizes all major viral envelope and capsid proteins (Card and Enquist, 1994). Subsequently, neurons were visualized with affinitypurified biotinylated donkey anti-rabbit IgG (1:2000; Jackson ImmunoResearch) and horseradish peroxidase reaction product (as described above) without nickel enhancement. In experiments in which two viral recombinants were injected, EGFP (produced by PRV-152) was detected using rabbit anti-GFP IgG (Molecular Probes, Eugene, OR) at 1:1000, $1: 200$, or 1:250, whereas $\beta$-gal (expressed by PRV-BaBlu) was detected with mouse anti- $\beta$-gal IgG (Sigma, St. Louis, MO) at 1:1500 or 1:5000. Primary antibodies were combined in a mixture at the concentrations described above together with $0.3 \%$ Triton X-100 and $1 \%$ normal donkey serum, and tissue was incubated in this solution for $48-72 \mathrm{hr}$ at $4^{\circ} \mathrm{C}$. Subsequently, tissue was placed for $2 \mathrm{hr}$ in the dark into a secondary antibody solution containing donkey anti-mouse IgG conjugated to Cy3 (1:200; Jackson ImmunoResearch) and goat anti-rabbit IgG conjugated to BODIPY-FL (1:200; Molecular Probes).

Specificity of the primary and secondary antibodies used in our double-labeling experiments has been documented previously (Billig et al., 2000). Additionally, in two rats the specificity of the antibodies was verified further. These animals were injected with $30 \mu \mathrm{l}$ of PRV-BaBlu into the gastrocnemius muscle on one side and $30 \mu \mathrm{l}$ of PRV-152 into the muscle on the other side. As expected, Cy3 and BODIPY-FL fluorescence signals were confined to the ventral horn of the lumbar spinal cord ipsilateral to each injection.

\section{Tissue analysis}

Tissue was examined using a Leica DMRD photomicroscope. The rat atlas of Paxinos and Watson (1986) was used as a reference for anatomical classification of various brainstem areas, and a study by Molander and colleagues (Molander et al., 1984) was used to guide the spinal cord tissue analysis.

Infected neurons were digitized using a Sony DXC-970MD color camera and MCID Elite 6.0 imaging software (Imaging Research, St. Catharines, Ontario). Different color fluorophores were detected using specific filter sets (Chroma Technology, Brattleboro, VT) with the following respective excitation and emission ranges: FG, 300-400 nm and $440-$ $490 \mathrm{~nm}$ (blue fluorescence); BODIPY-FL, 460-500 nm and 510-560 nm (green fluorescence); Cy3, 560-680 nm and 660-740 nm (red fluorescence). Use of such specific filter sets ensured that we did not detect any false-positive results. Images of neurons where obtained using each of the filter sets individually, and the presence of multiple fluorophores within a given cell was determined by digitally overlaying the images. Brightness and contrast of images were optimized for presentation purposes, whereas color balance and content were not altered in any of the photomicrographs. Digital images were imported into Canvas 6.0 (Deneba Systems, Miami, FL) and Photoshop 6.0 (Adobe Systems, San Jose, CA) software for preparation of figures.

\section{Statistical analysis}

Linear regression analyses of data were performed using Microsoft (Redmond, WA) Excel 2002 Software for Windows. Significance level was set as $p<0.05$.

\section{Results \\ Spinal cord labeling}

\section{Identification of gastrocnemius motoneurons}

The location of gastrocnemius motoneurons was determined in two rats by retrograde transport of CT $\beta$. Tissue harvested from the rats was cut in the coronal plane at $50 \mu \mathrm{m}$. One of four sections was processed for visualization of $\mathrm{CT} \beta$; thus the sections were $\sim 200 \mu \mathrm{m}$ apart. Gastrocnemius motoneurons were located within the lateral ventral horn on the side ipsilateral to the injection site. The neurons were confined to a column within lamina 9 at the L4 and L5 spinal levels. As illustrated in Figure $1 A, \mathrm{CT} \beta$ label was distributed throughout the somata (diameters of $\sim 40-70 \mu \mathrm{m})$ and also filled the proximal dendrites of the mo- 

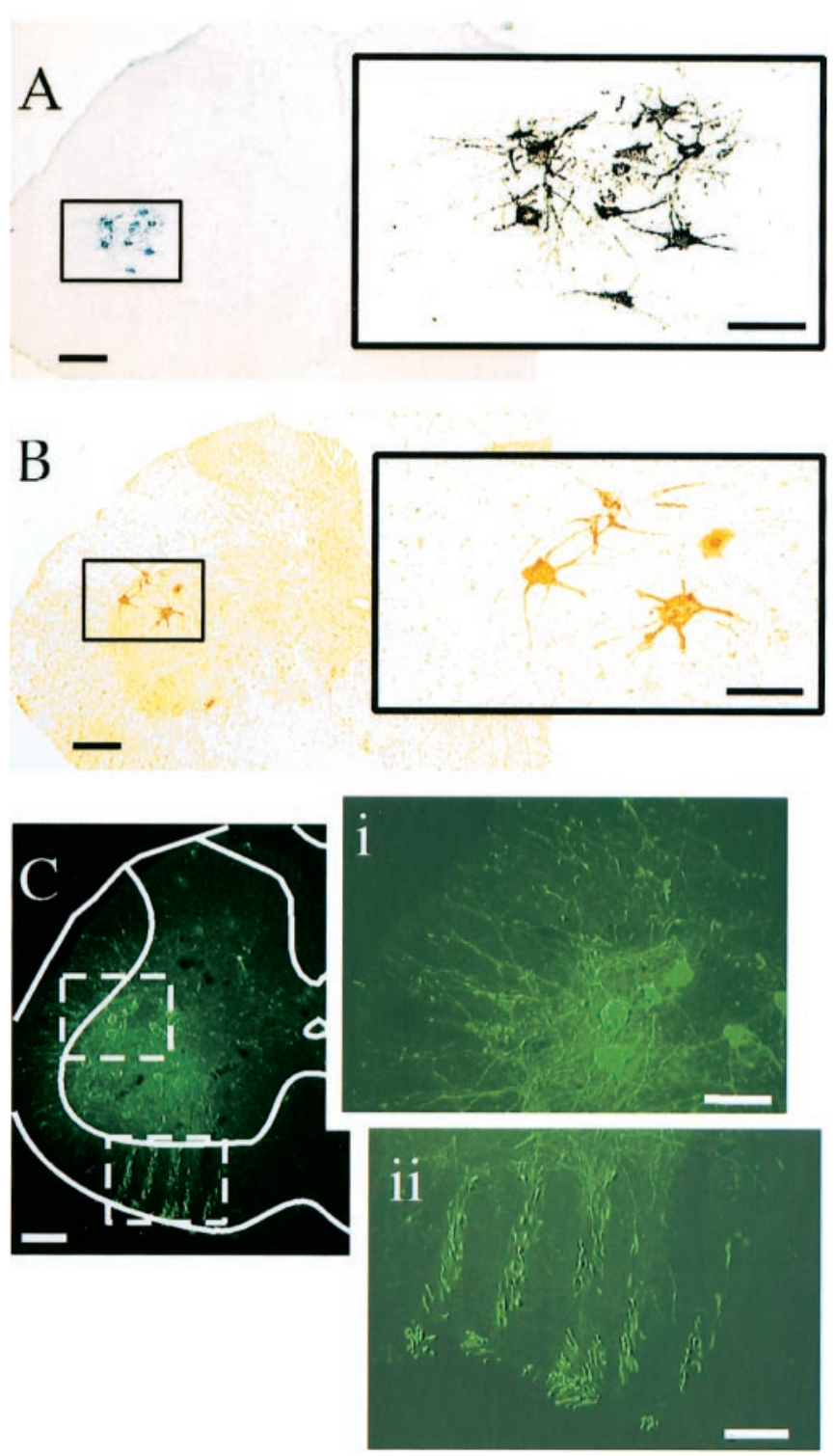

Figure 1. Labeling of gastrocnemius motoneurons produced by injecting $\mathrm{CT} \beta(A)$ or PRV ( $B$, $C)$ into the left gastrocnemius muscle. Under a low-power objective $(A)$ it was evident that labeled neurons were confined to a tight column within lamina IX of the L 4 and $L 5$ spinal cord levels. At higher magnification (inset to the right), it was observed that presumed gastrocnemius motoneurons had a soma diameter of $\sim 40-70 \mu \mathrm{m}$ and extensive dendritic arborizations. Billustrates the earliest neurons infected with PRV after its injection into the left gastrocnemius muscle. These neurons were visualized with the immunoperoxidase method and were of similar size and located at the same spinal levels as those labeled by $\mathrm{CT} \beta(A)$. Gastrocnemius motoneuron labeling was also achieved with an immunofluorescent technique ( $C$. The sensitivity of the technique is evident in the fact that PRV immunofluorescence was detected within somata and dendritic arborizations of motoneurons (i) as well as within axons (ii). Scale bars: low-power images $(A-C), 200 \mu \mathrm{m}$; high-power images (insets), $100 \mu \mathrm{m}$.

toneurons. A total of 126 motoneurons were labeled in the first animal (an average of 5.5 motoneurons per section), and 73 motoneurons were labeled in the second animal (an average of 7.3 motoneurons per section). It is likely that these numbers represent near maximal labeling of the gastrocnemius motor pool because CT $\beta$ was injected at multiple sites along the entire extent of the muscle, and these values are in agreement with those from previous investigations in the rat (Hashizume et al., 1988; Kanda et al., 1989).

In subsequent experiments, different numbers of $1 \mu \mathrm{l}$ injec- tions of PRV-152 were made into the left gastrocnemius muscle to determine the total injection volume required to produce a consistent infection of the entire motoneuron pool. The total volumes of PRV injected were as follows: $10 \mu \mathrm{l}$ ( $n=1 \mathrm{rat}), 15 \mu \mathrm{l}$ $(n=1), 20 \mu \mathrm{l}(n=2)$, and $30 \mu \mathrm{l}(n=7)$. Figure $1 B$ illustrates examples of motoneurons infected by injection of PRV-152 into gastrocnemius that were visualized using immunoperoxidase, whereas Figure $1 C$ shows infected motoneurons that were labeled using immunofluorescence. As indicated in Figure $2 \mathrm{~A}$, when 30 $\mu l$ injection volumes were used a reliable infection of gastrocnemius motoneurons was achieved; smaller volumes were less effective in producing labeling. There was also a strong negative correlation between the weight of the animal and the extent of motoneuron infection in the lumbar spinal cord. Figure $2 B$ illustrates that animals weighing $<300$ gm had the most robust infections of gastrocnemius motoneurons. Conversely, infection of a large number of gastrocnemius motoneurons was less likely in animals that weighed $\geq 400 \mathrm{gm}$. For example, the largest animal used in this experiment (476 gm) exhibited only a few labeled motoneurons after injection of $30 \mu \mathrm{l}$ of PRV into gastrocnemius (Fig. 2A, arrow). Importantly, the number of motoneurons labeled per section after $30 \mu$ injections of PRV in animals weighing $<300$ gm (average of $5.6 \pm 0.6$, SD) was similar to the number of motoneurons labeled after CT $\beta$ injections (mean of 5.5 and 7.3 motoneurons per section in the two rats used in this analysis). Collectively, these data suggest that we were able to infect the vast majority of the gastrocnemius motoneuron pool with PRV-152 without spread of virus to adjacent muscle groups.

To evaluate the effectiveness of the hindlimb sympathectomy, thoracic and upper lumbar spinal cord sections were examined for the presence of IML neurons infected by PRV-152. In shamoperated animals, large numbers of thoracic and lumbar IML neurons were infected with PRV-152. Labeling of small and medium neurons was also present near the central canal in laminas 7 and 10 of upper lumbar spinal segments. Additionally, in shamsympathectomized animals, considerable numbers of large neurons were observed within laminas 8 and 9 of the upper lumbar spinal cord segments, rostral to the gastrocnemius motor pool. These presumed motoneurons were apparently infected via direct or multisynaptic connections with IML neurons because this labeling was absent in sympathectomized rats. Furthermore, similar ventral horn labeling was observed after PRV-BaBlu injections into the adrenal gland, suggesting the existence of intraspinal connections between motor and sympathetic outflows.

\section{Labeling of SPN and interneurons}

To determine the location of SPNs in horizontal spinal cord sections, the retrograde tracer FG was injected intraperitoneally (Joshi et al., 1995). In three animals FG injections were combined with injections of PRV-152 into the left gastrocnemius muscle and injections of PRV-BaBlu into the left adrenal gland. Figure 3 illustrates the location of SPNs innervating the adrenal gland, which were double labeled with FG and PRV-BaBlu. Consistent with previously published reports (Strack et al., 1989; Li et al., 1992; Joshi et al., 1995; Edwards et al., 1996), adrenal SPNs were scattered throughout the IML, from the T2 to L2 spinal levels, with the greatest numbers detected at the mid-thoracic levels. As shown in Figure 3, we also observed a population of PRV-BaBlu immunofluorescent IML neurons that did not contain FG. It is possible that these cells were presympathetic interneurons infected transneuronally from adrenal SPNs. Additionally, a population of IML neurons was labeled for the presence of FG but not 

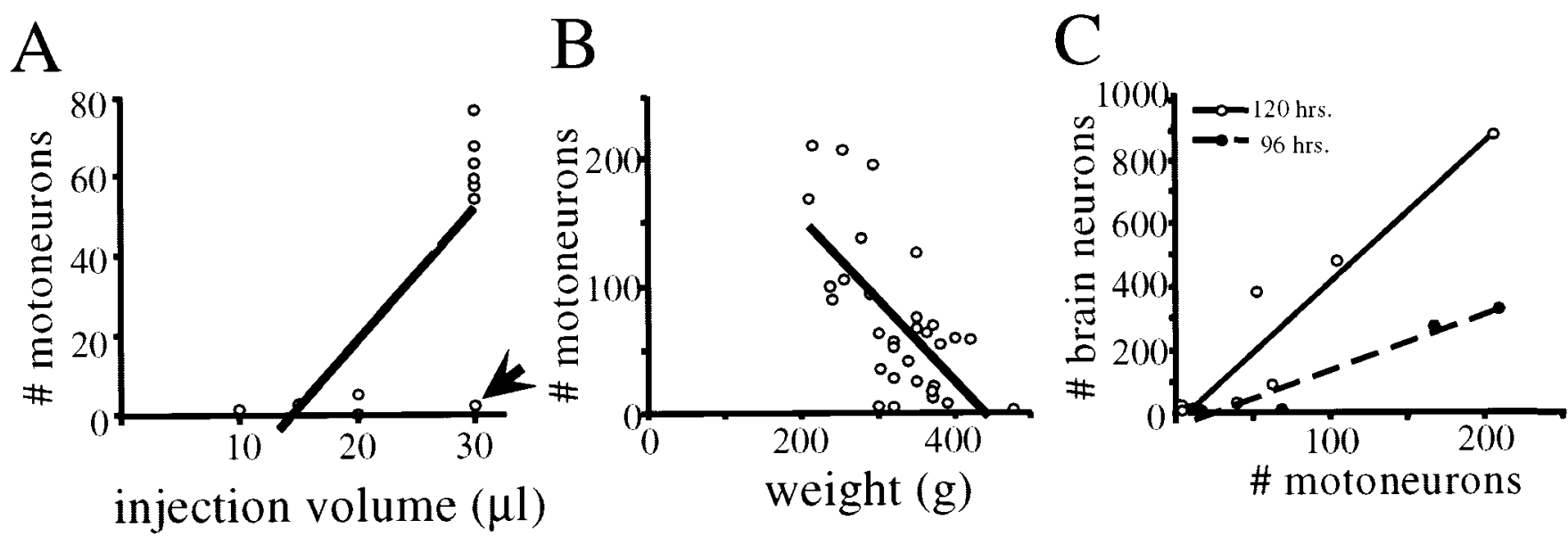

Figure 2. Factors influencing the infection of motoneurons and the subsequent transneuronal infection of brainstem neurons after injection of PRV-152 into the gastrocnemius muscle. $A$, Effect of increasing the total volume of virus injected on the number of motoneurons infected. In general, injection of $30 \mu$ l of PRV consistently produced infection of a substantial number of motoneurons; the only exceptional case (identified by arrow) was the largest animal (weight, $476 \mathrm{gm}$ ) used in this study. A linear regression revealed a significant relationship between the volume of virus injected and the number of infected motoneurons $\left(r^{2}=0.58 ; p=0.01\right)$; the regression line is included in the figure. B, Astrong negative correlation was also noted between the animal's weight and the total number of motoneurons infected $\left(r^{2}=\right.$ $0.41 ; p<0.001)$. C, The total number of brainstem neurons infected with PRV-152 was correlated directly with the number of gastrocnemius motoneurons infected. This linear correlation had a steeper slope in animals surviving $120 \mathrm{hr}$ after viral inoculations $\left(r^{2}=0.89 ; p<0.01\right)$ as compared with those killed at $96 \mathrm{hr}\left(r^{2}=0.93 ; p<0.01\right)$.
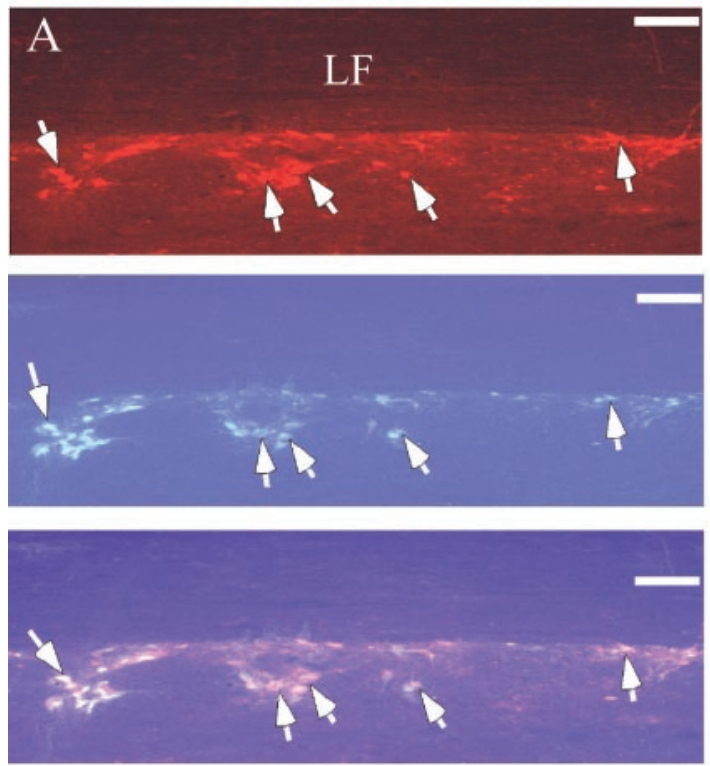
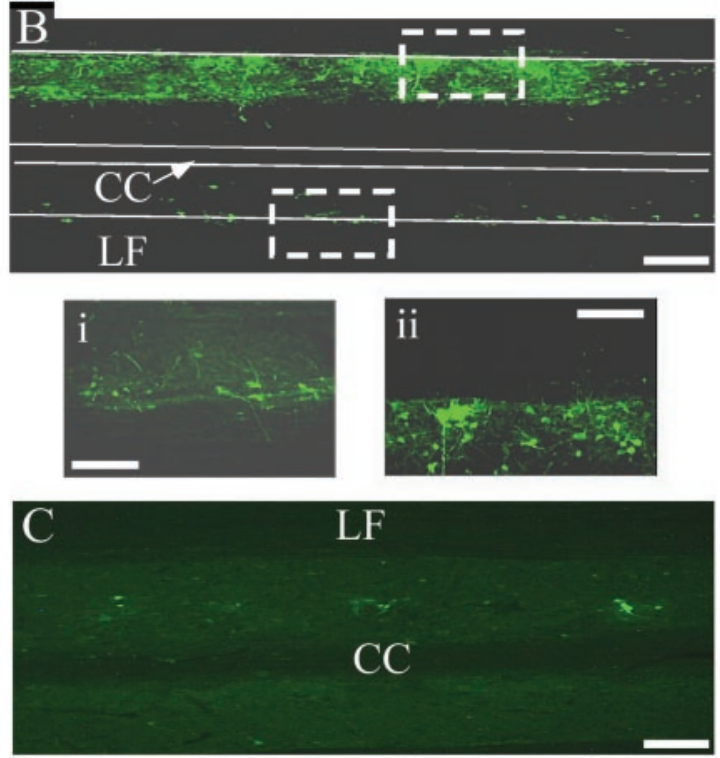

Figure 3. Identification of SPNs in horizontal sections of the T5-T7 spinal cord. A, Top, SPNs infected with PRV-BaBlu were visualized with Cy3-conjugated antibodies and appeared red. These neurons were observed at their expected location in the IML at the junction of the gray matter and the lateral funiculus (LF). $A$, Middle, Systemic injections of $\mathrm{FG}$ were made in the same animal to identify all of the sympathetic preganglionic neurons. FG, a blue-emitting fluorophore, was visualized with a specific UV fluorescence filter. $A$, Bottom, When immunofluorescences of both $\mathrm{FG}$ and Cy3 were overlaid, a subset of cells was identified as containing both markers (indicated with arrows). These cells were classified as adrenal preganglionic neurons. Neurons containing Cy 3 but not FG fluorescence were most likely second-order neurons labeled through the trans-synaptic transmission of PRV from SPNs, whereas those neurons containing only FG were classified as SPNs projecting to non-adrenal targets. IML neurons were also infected with PRV-152 after its injection into the gastrocnemius muscle of sham-sympathectomized rats ( $B$ ). Most of these cells were located ipsilateral to the injected muscle (Bii), although some infected neurons were also observed contralaterally (Bi). In contrast, PRV-152 infection of IML neurons was absent in sympathectomized rats (C). Scale bars: low-power images, $250 \mu \mathrm{m}$; high-power images (insets), $200 \mu \mathrm{m}$. CC, Central canal; LF, lateral funiculus.

PRV-BaBlu, indicating that adrenal SPNs are intermingled with SPNs providing innervation to other visceral organs.

\section{Brainstem labeling}

The animals that received PRV-152 injections into the left gastrocnemius muscle and PRV-BaBlu injections into the ipsilateral adrenal gland were divided into three groups on the basis of the success of the hindlimb sympathectomy. Group 1 rats $(n=7)$ received a sham sympathectomy and were used as negative controls. Animals in group $2(n=12)$ received a successful hindlimb sympathectomy, as indicated by a lack of neurons immunopositive for PRV-152 in the IML along with the presence of a similar number of infected motoneurons as in group 1 rats. Rats in group $3(n=8)$ were those in which the sympathectomy resulted in only a partial reduction of PRV-152-containing IML neurons. Because infection of sympathetic efferents with PRV injected into gastrocnemius would confound interpretation of our data, these animals were excluded from further analysis, and the data will not be discussed.

Of the 12 group 2 (successful sympathectomy) animals, 5 were 

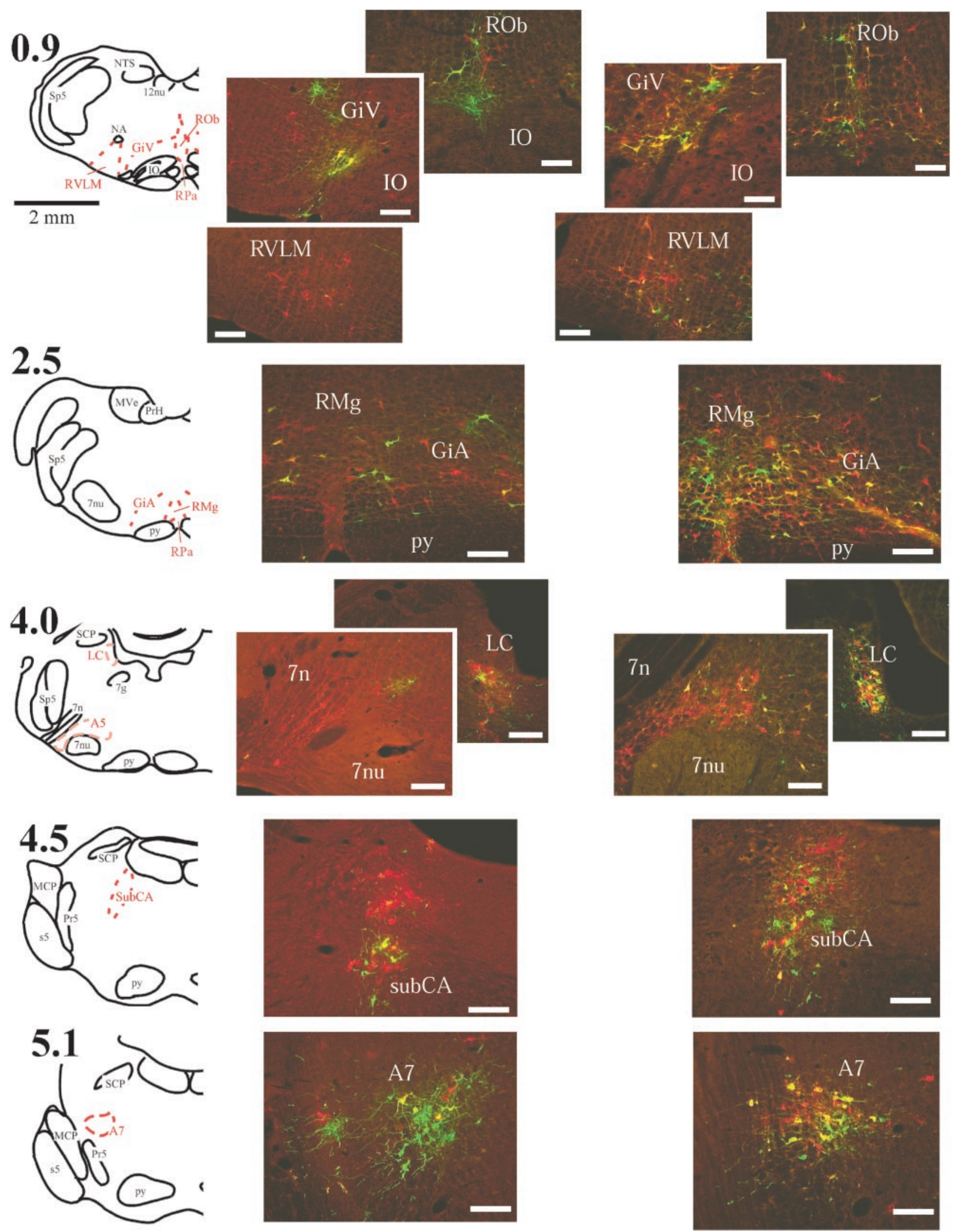

Figure 4. Location of double-labeled brainstem neurons. Medullary and pontine neurons infected with only one of the viral recombinants were identified with either green-emitting (PRV-152) or red-emitting (PRV-BaBlu) fluorophores, whereas neurons that contained both recombinants appeared yellow. Drawings in the left column indicate the locations of anatomical regions shown to the right. Numbers next to the drawings represent the approximate distance in millimeters from calamus scriptorius, defined as the point of convergence of the fourth ventricle to form the central canal. Photomicrographs located in the middle column (immediately to the right of the drawings) represent data from an animal allowed to survive $96 \mathrm{hr}$ after injection, whereas those on the far right were taken from an animal that survived $120 \mathrm{hr}$ after PRV-152 injection into the left gastrocnemius muscle and $96 \mathrm{hr}$ after PRV-BaBlu injection into the left adrenal gland. Note the increase in the numbers of double-labeled neurons after the longer survival time. Scale bars, $200 \mu \mathrm{m}$. 7g, Facial nerve genu; 7n, facial nerve; 7nu, facial nucleus; 12nu, hypoglossal nucleus; GiA, 

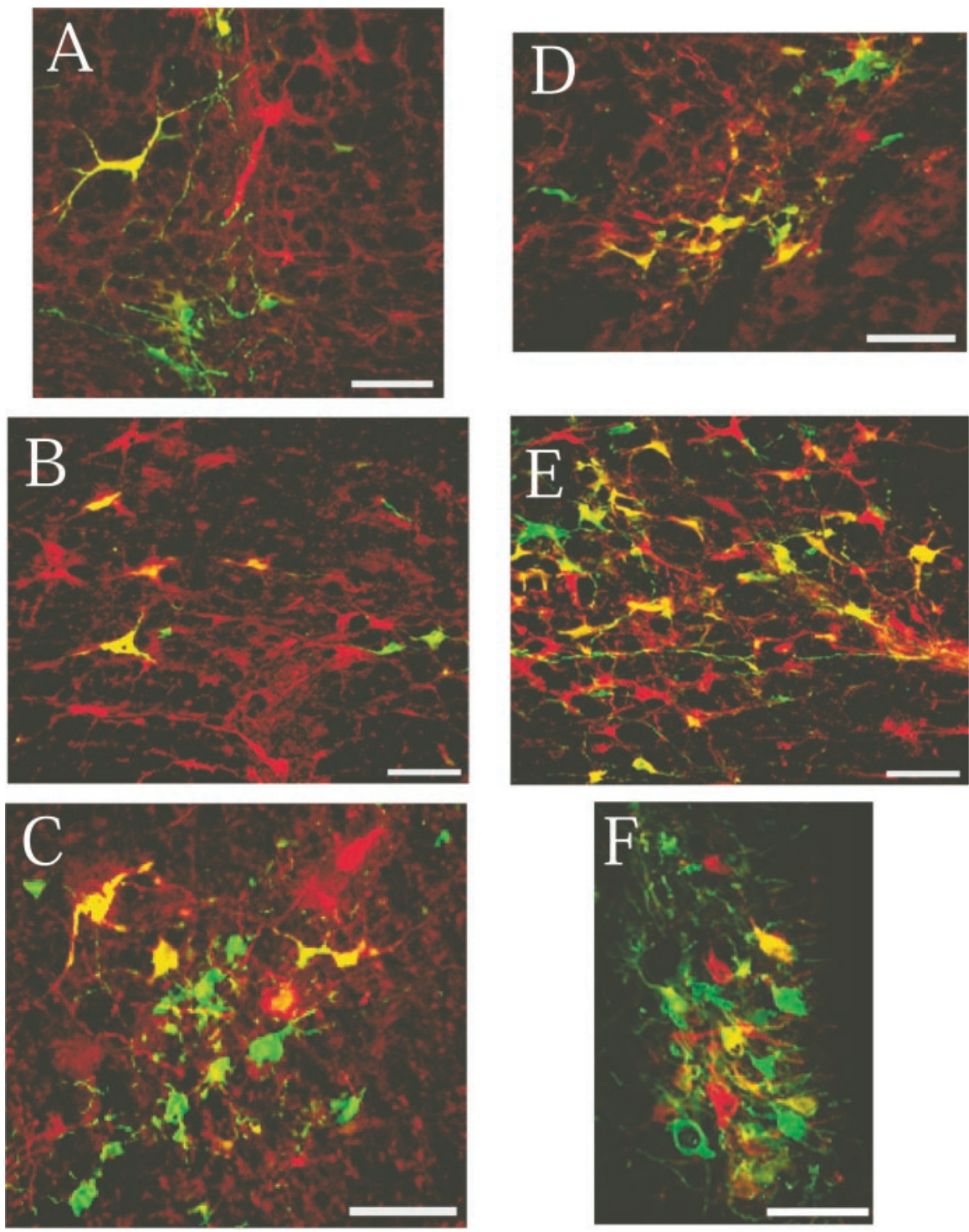

Figure 5. High-power photomicrographs of regions containing substantial numbers of infected neurons at different pontomedullary levels. $A-C$, Images taken from an animal that survived $96 \mathrm{hr}$ after viral injection into the left gastrocnemius muscle and the left adrenal gland; $D-F$, images taken from an animal that survived $120 \mathrm{hr}$ after PRV-152 injection into the left gastrocnemius muscle and $96 \mathrm{hr}$ after PRV-BaBlu injection into the left adrenal gland. Labeling is shown in the following regions: $A$, raphe obscurus; $B$, raphe magnus; $C, A 7 ; D$, ventral gigantocellular nucleus; $E$, gigantocellular nucleus pars alpha; $F$, locus coeruleus. Scale bars, $100 \mu \mathrm{m}$.

euthanized $96 \mathrm{hr}$ after the injection of PRV into both the adrenal gland and the gastrocnemius muscle, and the remaining 7 survived $120 \mathrm{hr}$ after the gastrocnemius injection and $96 \mathrm{hr}$ after the adrenal injection. In the latter animals, injections into gastrocnemius and the adrenal gland were separated by $24 \mathrm{hr}$ to balance the temporal pattern of brainstem infection with both recombinants. Previous work has demonstrated that infection of a neuron with one viral strain can preclude subsequent infection with another recombinant (Kim et al., 1999). Thus, temporal matching in the transmission of the two recombinants maximized the chances for their simultaneous expression within the same cell.
The initial analysis focused on parameters influencing transmission of PRV-152 to the brainstem. As illustrated in Figure $2 C$, the number of PRV-152-infected brainstem neurons was correlated significantly with the number of gastrocnemius motoneurons that were infected with this recombinant at both the $120 \mathrm{hr}\left(r^{2}=0.89\right.$; $p<0.01)$ and the $96 \mathrm{hr}\left(r^{2}=0.93 ; p<\right.$ $0.01)$ survival periods. There appeared to be a threshold number of gastrocnemius motoneurons that were required to be infected with PRV-152 before appreciable labeling was observed in the brainstem. For the $120 \mathrm{hr}$ survival period this number was $\sim 50$ gastrocnemius motoneurons, whereas for the $96 \mathrm{hr}$ survival period this number was $>150$. Thus, the total number of PRV-152-positive neurons in the brainstem was greater after longer survival times as well as with an increased number of infected motoneurons.

Examination of the brainstem in sympathectomized and sham-operated rats revealed a consistent presence of neurons infected with one or both of the viral recombinants. These neurons were observed in the rostral ventrolateral medulla (RVLM), ventral gigantocellular nucleus (GiV), gigantocellular nucleus pars alpha (GiA), raphe pallidus (Rpa), raphe obscurus (Rob), raphe magnus (RMg), the A5 and A7 noradrenergic cell groups, locus coeruleus (LC), and subcoeruleus (subCA). The numbers of neurons infected with PRV-152 in the RVLM was much greater in sham than in sympathectomized animals. However, both the total number of PRV-152-positive brainstem neurons and the number of double-infected neurons in particular brainstem regions differed among animals. Of the 12 animals with complete hindlimb sympathectomies, 5 displayed double-labeled neurons in RVLM, 10 in $\mathrm{GiV}, 7$ in ROb, 5 in RPa, $6 \mathrm{RMg}, 8$ in GiA, 6 in A5, 7 in LC, 6 in subCA, and 8 in A7. The most prominent source of this variability appeared to be infection of an insufficient number of gastrocnemius motoneurons to produce transneuronal spread of virus to the brainstem. This factor could be controlled for in six animals in which $>50$ gastrocnemius motoneurons were infected at $120 \mathrm{hr}$ after injection or $>150$ gastrocnemius motoneurons were infected at $96 \mathrm{hr}$ after injection. All six of these animals exhibited double-infected neurons within GiV, GiA, ROb, RMg, LC, subCA, and A7. In addition, five of these rats displayed doublelabeled neurons in RVLM, RPa, and A5 (Fig. 4).

The presence of double labeling was confirmed by examining the tissue under illumination that excited each of the fluoro- 


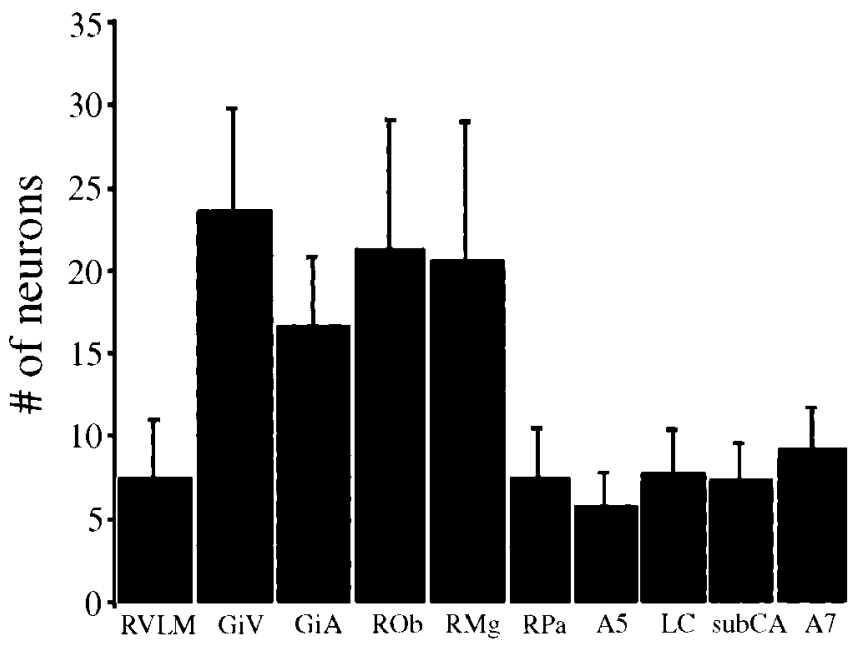

Figure 6. Mean number of neurons coinfected with both PRV-152 and PRV-BaBlu in different brainstem regions. The number of neurons infected with both recombinants was quantified in 10 medullary and pontine regions of 12 rats in which the hindlimb was surgically sympathectomized, and PRV-152 was injected into the gastrocnemius muscle, whereas PRV-BaBlu was injected into the ipsilateral adrenal gland. Numbers represent total number of double-labeled cells (quantified across multiple sections) in each region; see Results for details of the quantification procedure. Double-labeled neurons were distributed bilaterally but were slightly more prevalent ipsilateral to injections; labeling on both sides was counted to quantify the distribution of infected cells. GiA, Gigantocellular nucleus pars alpha; GiV, ventral gigantocellular nucleus; LC, locus coeruleus; RMg, raphe magnus; ROb, raphe obscurus; RPa, raphe pallidus; RVLM, rostral ventrolateral medulla; subCA, nucleus subcoeruleus.

phores alone, to ensure that the labeling was not caused by the overlapping of two cells that were each infected by a single viral recombinant. Infected neurons contained viral immunoreactivity in cell nuclei and cytoplasm. Viral antigen in the somatodendritic compartment also extended into dendrites to varying degrees (Fig. 5). The tissue also included fluorescent signal representing portions of neurons and processes that were not clearly connected to a cell body within the same section. Such neuronal fractions were excluded from further analyses.

The number of double-infected neurons in different anatomical locations was determined in the six animals with the most extensive motoneuron labeling. Cells were counted only if the soma and processes were clearly defined. Furthermore, care was taken to analyze the same number of coronal brainstem sections in each animal; the sections were cut at $40 \mu \mathrm{m}$ thickness, and every sixth section was examined (spacing between sections of $240 \mu \mathrm{m})$. As shown in Figure 4, the RVLM was defined as the triangular region at the ventral surface of the brainstem ventral to nucleus ambiguus and lateral to the inferior olive. Four consecutive sections were used to quantify RVLM labeling, with the caudalmost section located $\sim 480 \mu \mathrm{m}$ rostral to calamus scriptorius, defined as the point at which the fourth ventricle converges to form the central canal. GiV was defined as the area immediately dorsal to the inferior olive and bounded laterally by the medial extent of the RVLM and medially by the raphe nuclei; four sections were used to quantify GiV labeling, with the caudalmost section located $\sim 240 \mu \mathrm{m}$ rostral to calamus scriptorius. Four sections were used to determine cell numbers within the caudal raphe cell groups: $\mathrm{ROb}, \mathrm{RPa}$, and $\mathrm{RMg}$. GiA labeling was counted in four sections located immediately rostral to $\mathrm{GiV}$, in an area that extended ventrolaterally from the portion of the midline dorsal to RMg. Three sections per animal were used to quantify labeling in LC and subCA. The A7 cell group was defined as the area located between the ventrolateral pole of the superior cere- bellar peduncle at its intermediate rostrocaudal position and the dorsomedial tip of the trigeminal sensory nucleus and tract. Neuronal labeling in this cell group was quantified in two consecutive sections. A5 was defined as a triangle with the ventral edge of the brainstem along the lateral and inferior aspect, the root of the seventh nerve along the lateral and superior aspect, and the inferior olive located medially. The number of double-labeled neurons was determined in four sections from A5.

Double-labeled neurons were distributed bilaterally but were slightly more prevalent ipsilateral to injections; labeling on both sides was counted to quantify the distribution of infected cells. The number of double-labeled neurons appeared to be greater within the ventromedial medullary areas, GiV, GiA, ROb, and $\mathrm{RMg}$, than in the other regions (Fig. 6).

\section{Discussion}

This study exploited the ability of PRV to infect chains of synaptically linked neurons and is the first direct examination of the locations of CNS neurons that innervate both sympathetic and motor outflows. It seems likely that these neurons play a role in concomitant regulation of sympathetic and motor efferent activities during behaviors that require such coordination, such as exercise. Our data show that neurons that participate in coordinated motor and autonomic regulation exist in several brain regions but are concentrated in the dorsolateral pons (A7, LC, and subCA), the rostral ventromedial medulla (raphe nuclei, GiV, and GiA), and the lateral brainstem (RVLM and the A5 cell group).

Among the areas described above, the region of the rostral ventromedial medulla near the pontomedullary junction contained the greatest numbers of neurons that were double-infected with the viral recombinants injected into both the adrenal gland and the gastrocnemius muscle. In addition, double-infected neurons were detected in this region at the shortest survival times that resulted in brainstem labeling, suggesting that these doublelabeled cells are part of the reticulospinal projection system and that their axons likely collateralize to innervate both adrenal SPNs and gastrocnemius motoneurons. This finding is supported by previous studies using monosynaptic tracers that demonstrated extensive, collateralized projections from the rostral ventromedial medulla to the spinal cord in various species (Martin et al., 1979a; Zemlan et al., 1984; Martin et al., 1985; Holstege and Kuypers, 1987). Furthermore, Allen and Cechetto (1994) injected fluorescent retrograde tracers into the rat ventral horn as well as the IML to ascertain whether reticulospinal neurons made connections with both SPNs and motoneurons and demonstrated that a small population of cells in the rostral ventromedial medulla fit this criterion. However, their data were confounded by the fact that tracer spread occurred at the injection sites such that non-target neurons may have been labeled. In contrast, trans-synaptic labeling of motor and autonomic pathways in the present study showed that a substantial number of rostral ventromedial medulla neurons provide inputs to both motoneurons and SPNs.

The dorsolateral pons also contained neurons that were dually infected by the viral recombinants injected into gastrocnemius and the adrenal gland, although the cells were less numerous than those in the rostral ventromedial medulla. This finding is not unexpected, because studies using monosynaptic tracers have suggested that LC, subCA, and the A7 cell group neurons, including noradrenergic cells located in these regions, have collateralized projections to the spinal cord (Nygren and Olson, 1977; Holstege et al., 1979; Martin et al., 1979b; Jones and Yang, 1985; 
Holstege and Kuypers, 1987; Lyons and Grzanna, 1988). Some double-infected neurons were also observed in the A5 cell group and the RVLM, which is surprising because projections from these regions are believed to terminate mainly within the IML (Loewy et al., 1979, 1981; Byrum and Guyenet, 1987; Loewy, 1990). These findings cannot be explained by the infection of sympathetic fibers innervating hindlimb targets (e.g., vascular smooth muscle) because labeling was observed in the RVLM and A5 cell group in animals in which the hindlimb was completely sympathectomized, as evidenced by the lack of PRV-152-infected IML neurons. However, because double labeling of cells in these areas was limited to the longer survival times used in the study, it is possible that it represents the passage of virus through multisynaptic pathways instead of through collateralized projections to both adrenal SPNs and gastrocnemius motoneurons.

The number of motoneurons infected by injection of PRV152 into the gastrocnemius muscle as well as the number of brainstem neurons trans-synaptically infected by this virus varied inversely with an animal's weight. One possibility is that this effect was caused by the size of the muscle. Presumably the gastrocnemius muscle enlarges as the animal gets older and heavier, thus dispersing motor end-plates and motoneuron terminals over a greater area. This could effectively decrease the viral titer at each motoneuron terminal, thereby decreasing the probability of PRV entry into a particular terminal. An alternative explanation of this finding is that it may be related to the animal's age: younger animals may be more susceptible to infection with PRV. Consistent with this notion, it has been reported that swine are most vulnerable to developing Aujezsky's disease after exposure to PRV when they are young (Gustafson, 1975). Others have also suggested a relationship between an animal's age and the robustness of CNS infection after PRV inoculation (Cano, 2002). Further study is required to definitively address this issue and to determine the mechanism through which aging may limit the ability for a neuron to be infected by PRV.

Although this study revealed that neurons in several brainstem regions, most prominently the rostral ventromedial medulla, are likely to participate in both autonomic and motor control, available data do not permit a precise definition of the hierarchy of connections. This is attributable in large part to the demonstration that the onset of viral replication within a circuit is connection dependent (Card et al., 1999). Thus, the progression of infection through a polysynaptic pathway depends on the density of synaptic connections of neurons within a circuit, and hierarchy cannot be determined solely on the basis of postinoculation survival interval. For this reason, it is unclear whether neurons in the A5 cell group and RVLM provide direct or indirect inputs to both adrenal SPNs and gastrocnemius motoneurons. Furthermore, because of this limitation we did not extend the survival times sufficiently to permit infection of neurons in the cerebral cortex or diencephalon, because it would be very difficult to conclude whether labeling produced at this long interval was caused by transport of virus through direct or multisynaptic projections from these regions to the IML and lumbar ventral horn. The possibility thus remains that areas rostral to the pons, which were not identified in this study, participate directly in coordinated autonomic and motor control. In addition, because infection of a neuron with one virus makes it less susceptible to infection by a second virus (Kim et al., 1999), it is feasible that the number of neurons providing inputs to both SPNs and motoneurons was underestimated in some brain regions. This possibility was minimized by injecting PRV into the gastrocnemius muscle and the adrenal gland on different days in some animals to bal- ance the latency at which the two viruses arrived at the brainstem, but it cannot be discounted completely.

Brainstem neurons with projections to both SPNs and motoneurons could play a number of different functional roles. These cells could serve to simultaneously adjust the excitability of motor and autonomic output pathways, so that both pathways would be more or less responsive to specific commands relayed through other neural circuits. For example, inhibitory bulbospinal neurons could potentially globally reduce both autonomic and motor activity during behaviors such as sleep (Futuro-Neto and Coote, 1982). Alternately, subsets of brainstem neurons could serve to coordinate specific activities that involve simultaneous contractions of certain muscles and alterations in firing of particular sympathetic efferents, such as sexual behaviors including lordosis (Zemlan et al., 1983). Thus, further experiments are warranted to explore the role of neurons in the rostral ventromedial medulla in eliciting coordinated motor and autonomic responses.

\section{References}

Allen GV, Cechetto DF (1994) Serotoninergic and nonserotoninergic neurons in the medullary raphe system have axon collateral projections to autonomic and somatic cell groups in the medulla and spinal cord. J Comp Neurol 350:357-366.

Benarroch EB (1993) The central atonomic network: functional organization, dysfunction, and perspective. Mayo Clin Proc 68:988-1001.

Billig I, Foris JM, Card JP, Yates BJ (1999) Transneuronal tracing of neural pathways controlling an abdominal muscle, rectus abdominis, in the ferret. Brain Res 820:31-44.

Billig I, Foris JM, Enquist LW, Card JP, Yates BJ (2000) Definition of neuronal circuitry controlling the activity of phrenic and abdominal motoneurons in the ferret using recombinant strains of pseudorabies virus. J Neurosci 20:7446-7454.

Billig I, Hartge K, Card JP, Yates BJ (2001) Transneuronal tracing of neural pathways controlling abdominal musculature in the ferret. Brain Res 912:24-32.

Byrum CE, Guyenet PG (1987) Afferent and efferent connections of the A5 noradrenergic cell group in the rat. J Comp Neurol 261:529-542.

Cannon WB (1963) The wisdom of the body. New York: W. W. Norton.

Cano G (2002) Characterization of the CNS control using viral transneuronal tracing. PhD thesis, University of Pittsburgh.

Cano G, Card JP, Rinaman L, Sved AF (2000) Connections of Barrington's nucleus to the sympathetic nervous system in rats. J Auton Nerv Syst 79:117-128.

Card JP, Rinaman L, Lynn RB, Lee BH, Meade RP, Miselis RR, Enquist LW (1993) Pseudorabies virus infection of the rat central nervous system: ultrastructural characterization of viral replication, transport, and pathogenesis. J Neurosci 13:2515-2539.

Card JP, Enquist LW (1994) The use of neurotropic herpes viruses for defining synaptically linked populations of neurons in the central nervous system. In: Methods in molecular genetics, molecular biology techniques (Adolph KW, ed), pp 363-382. San Diego: Academic.

Card JP, Enquist LW, Moore RY (1999) Neuroinvasiveness of pseudorabies virus injected intracerebrally is dependent on viral concentration and terminal field density. J Comp Neurol 407:438-452.

Edwards SL, Anderson CR, Southwell BR, McAllen RM (1996) Distinct preganglionic neurons innervate noradrenaline and adrenaline cells in the cat adrenal medulla. Neuroscience 70:825-832.

Enquist LW, Husak PJ, Banfield BW, Smith GA (1998) Infection and spread of alpha herpes viruses in the nervous system. Adv Virus Res 51:237-347.

Futuro-Neto HA, Coote JH (1982) Changes in sympathetic activity to heart and blood vessels during desynchronized sleep. Brain Res 252:259-268.

Gandevia SC, Killian K, McKenzie DK, Crawford M, Allen GM, Gorman RB, Hales JP (1993) Respiratory sensations, cardiovascular control, kinaesthesia and transcranial stimulation during paralysis in humans. J Physiol 470:85-107.

Greene EC (1963) Anatomy of the rat. New York: Hafner.

Gustafson DP, ed (1975) Pseudorabies. Ames, Iowa: Iowa State Press.

Hashizume K, Kanda K, Burke RE (1988) Medial gastrocnemius motor nu- 
cleus in the rat: age-related changes in the number and size of motoneurons. J Comp Neurol 269:425-430.

Holstege G, Kuypers HG, Boer RC (1979) Anatomical evidence for direct brain stem projections to the somatic motoneuronal cell groups and autonomic preganglionic cell groups in cat spinal cord. Brain Res 171:329-333.

Holstege JC, Kuypers HG (1987) Brainstem projections to spinal motoneurons: an update. Neuroscience 23:809-821.

Hsu SM, Raine L, Fanger H (1981) Use of avidin-biotin-peroxidase complex $(\mathrm{ABC})$ in immunoperoxidase techniques: a comparison between $\mathrm{ABC}$ and unlabeled antibody (PAP) procedures. J Histochem Cytochem 29:577-580.

Jänig W, McLachlan EM (1992) Characteristics of function-specific pathways in the sympathetic nervous system. Trends Neurosci 15:475-481.

Jones BE, Yang TZ (1985) The efferent projections from the reticular formation and the locus coeruleus studied by anterograde and retrograde axonal transport in the rat. J Comp Neurol 242:56-92.

Joshi S, Levatte MA, Dekaban GA, Weaver LC (1995) Identification of spinal interneurons antecedent to adrenal sympathetic preganglionic neurons using trans-synaptic transport of herpes simplex virus type 1 . Neuroscience 65:893-903.

Kanda K, Sato H, Hashizume K, Yamada J (1989) The effects of blocking nerve conduction on retrograde HRP labeling of rat motoneuron. Neurosci Lett 99:153-156.

Kandel ER, Schwartz JH, Jessell TM (2000) Principles of neural science. New York: McGraw-Hill.

Kim JS, Enquist LW, Card JP (1999) Circuit-specific coinfection of neurons in the rat central nervous system with two pseudorabies virus recombinants. J Virol 73:9521-9531.

Langley JN (1921) The autonomic nervous system. Cambridge, UK: W. Heffer.

Li Y-W, Ding Z-Q, Wesselingh SL, Blessing WW (1992) Renal and adrenal sympathetic preganglionic neurons in rabbit spinal cord: tracing with Herpes simplex virus. Brain Res 573:147-152.

Loewy AD (1990) Central autonomic pathways. In: Central regulation of autonomic functions (Loewy AD, Spyer KM, eds), pp 89-103. New York: Oxford UP.

Loewy AD, McKellar S, Saper CB (1979) Direct projections from the A5 catecholamine cell group to the intermediolateral cell column. Brain Res 174:309-314.

Loewy AD, Wallach JH, McKellar S (1981) Efferent connections of the ventral medulla oblongata in the rat. Brain Res 228:63-80.

Lyons WE, Grzanna R (1988) Noradrenergic neurons with divergent projections to the motor trigeminal nucleus and the spinal cord: a double retrograde neuronal labeling study. Neuroscience 26:681-693.

Martin GF, Humbertson AO, Laxson C, Panneton WM (1979a) Evidence for direct bulbospinal projections to laminae IX, X and the intermediolateral cell column. Studies using axonal transport techniques in the North American opossum. Brain Res 170:165-171.

Martin GF, Humbertson Jr AO, Laxson LC, Panneton WM, Tschismadia I (1979b) Spinal projections from the mesencephalic and pontine reticular formation in the North American opossum: a study using axonal transport techniques. J Comp Neurol 187:373-399.

Martin GF, Vertes RP, Waltzer R (1985) Spinal projections of the gigantocellular reticular formation in the rat. Evidence for projections from different areas to laminae I and II and lamina IX. Exp Brain Res 58:154-162.
Matsukawa K, Mitchell JH, Wall PT, Wilson LB (1991) The effect of static exercise on renal sympathetic nerve activity in conscious cats. J Physiol 434:453-467.

McLean IW, Nakane PK (1974) Periodate-lysine-paraformaldehyde fixative. A new fixation for immunoelectron microscopy. J Histochem Cytochem 22:1077-1083.

Molander C, Xu Q, Grant G (1984) The cytoarchitectonic organization of the spinal cord in the rat. I. The lower thoracic and lumbosacral cord. J Comp Neurol 230:133-141.

Nygren LG, Olson L (1977) A new major projection from locus coeruleus: the main source of noradrenergic nerve terminals in the ventral and dorsal columns of the spinal cord. Brain Res 132:85-93.

Papka RE, McCurdy JR, Williams SJ, Mayer B, Marson L, Platt KB (1995) Parasympathetic preganglionic neurons in the spinal cord involved in uterine innervation are cholinergic and nitric oxide-containing. Anat Rec 241:554-562.

Paxinos G, Watson C (1986) The rat brain in stereotaxic coordinates, Ed 2. San Diego: Academic.

Rowell LB (1993) Human cardiovascular control. New York: Oxford UP.

Smith BN, Banfield BW, Smeraski CA, Wilcox CL, Dudek FE, Enquist LW, Pickard GE (2000) Pseudorabies virus expressing enhanced green fluorescent protein: a tool for in vitro electrophysiological analysis of transsynaptically labeled neurons in identified central nervous system circuits. Proc Natl Acad Sci USA 97:9264-9269.

Standish A, Enquist LW, Miselis RR, Schwaber JS (1995) Dendritic morphology of cardiac related medullary neurons defined by circuit-specific infection by a recombinant pseudorabies virus expressing betagalactosidase. J Neurovirol 1:359-368.

Strack AM, Sawyer WB, Platt KB, Loewy AD (1989) CNS cell groups regulating the sympathetic outflow to adrenal gland as revealed by transneuronal cell body labeling with pseudorabies virus. Brain Res 491:274-296.

Vahlne A, Nystrom B, Sandberg M, Hamberger A, Lycke E (1978) Attachment of herpes simplex virus to neurons and glial cells. J Gen Virol 40:359-371.

Vahlne A, Svennerholm B, Sandberg M, Hamberger A, Lycke E (1980) Differences in attachment between herpes simplex type 1 and type 2 viruses to neurons and glial cells. Infect Immun 28:675-680.

Verberne AJ, Lam W, Owens NC, Sartor D (1997) Supramedullary modulation of sympathetic vasomotor function. Clin Exp Pharmacol Physiol 24:748-754.

Victor RG, Secher NH, Lyson T, Mitchell JH (1995) Central command increases muscle sympathetic nerve activity during intense intermittent isometric exercise in humans. Circ Res 76:127-131.

Vissing J, Wilson LB, Mitchell JH, Victor RG (1991) Static muscle contraction reflexly increases adrenal sympathetic nerve activity in rats. Am J Physiol 261:R1307-1312.

Waldrop TG, Eldridge FL, Iwamoto GA, Mitchell JH (1996) Central neural control of respiration and circulation during exercise. In: Handbook of physiology, Sect 12: Exercise: regulation and integration of multiple systems (Rowell LB, Shepherd JT, eds), pp 333-380. New York: Oxford UP.

Zemlan FP, Kow LM, Pfaff DW (1983) Effect of interruption of bulbospinal pathways on lordosis, posture, and locomotion. Exp Neurol 81:177-194.

Zemlan FP, Behbehani MM, Beckstead RM (1984) Ascending and descending projections from nucleus reticularis magnocellularis and nucleus reticularis gigantocellularis: an autoradiographic and horseradish peroxidase study in the rat. Brain Res 292:207-220. 